\title{
Antibiotic growth promoters enhance animal production by targeting intestinal bile salt hydrolase and its producers
}

\author{
Jun Lin* \\ Department of Animal Science, The University of Tennessee, Knoxville, TN, USA
}

\section{Edited by:}

Robert Paul Hunter, Lilly, USA

Reviewed by:

Noton Kumar Dutta, Johns Hopkins

University, USA

Dmitri Debabov, NovaBay

Pharmaceuticals, USA

\section{*Correspondence:}

Jun Lin, Department of Animal

Science, The University of Tennessee, 2506 River Drive, Knoxville,

TN 37996-4574, USA

e-mail: jlin6@utk.edu
The growth-promoting effect of antibiotic growth promoters (AGPs) was correlated with the decreased activity of bile salt hydrolase (BSH), an intestinal bacteria-produced enzyme that exerts negative impact on host fat digestion and utilization. Consistent with this finding, independent chicken studies have demonstrated that AGP usage significantly reduced population of Lactobacillus species, the major BSH-producers in the intestine. Recent finding also demonstrated that some AGPs, such as tetracycline and roxarsone, display direct inhibitory effect on BSH activity. Therefore, BSH is a promising microbiome target for developing novel alternatives to AGPs. Specifically, dietary supplementation of BSH inhibitor may promote host lipid metabolism and energy harvest, consequently enhancing feed efficiency and body weight gain in food animals.

Keywords: antibiotic growth promoters, bile salt hydrolase, Lactobacilli

\section{INTRODUCTION}

Epidemiological studies have linked usage of antibiotic growth promoters (AGPs) to the emergence of antibiotic resistant bacteria (Wegener, 2003). Antibiotic-resistant bacteria as well as resistance determinants can therefore spread from animals to humans, compromising the effectiveness of antibiotics for treating human infections and posing a serious threat to public health (Wegener, 2003). For this reason, Denmark banned all AGPs in 1998 and European Union member nations banned all AGPs in 2006 (Wegener, 2003; Dibner and Richards, 2005). Therefore, there is a worldwide trend to limit AGP use in food animals (Turnidge, 2004; Dibner and Richards, 2005). Ending the use of AGPs creates challenges for the animal feed and feed additive industries. Several products, such as probiotics, prebiotics, and organic acids have been used to alter intestinal microbiota for improving animal health and production (Dibner and Richards, 2005). However, limited data is available to justify the choice of specific bacterial species or products for such microbiota manipulation. Examination of microbiota in response to AGP treatment would provide insights into the modes of action of AGPs and facilitate the development of more effective microbiota-based strategies for growth promotion. Recent studies on the relationship between AGP usage and gut microbiota strongly suggest that bile salt hydrolase (BSH) is an important target through which microbiome composition and function may impact host fat digestion and energy harvest.

\section{RESPONSE OF INTESTINAL BSH ACTIVITY TO AGP}

Clearly a connection has been made between AGP usage, growth promotion, and intestinal bacterial populations, but the precise mechanism is yet to be delineated as to how these all coincide. Strides have been made in uncovering this mystery, however,

Abbreviations: AGP, antibiotic growth promoter; BSH, bile salt hydrolase; CBA, conjugated bile acid. and it has been shown that the growth-promoting effect of lowdose antibiotics does coincide with a decrease in BSH activity in the gut (Feighner and Dashkevicz, 1987; Knarreborg et al., 2004; Guban et al., 2006). BSH produced by gut bacteria catalyzes deconjugation of conjugated bile acids (CBAs, also referred to as conjugated bile salts) in the intestine (Begley et al., 2006). CBAs consist of a hydrophobic steroid core that is conjugated with either glycine or taurine. Thus, the CBAs are amphipathic and function as a more efficient "biological detergent" than unconjugated bile acids to emulsify and solubilize lipids for fat digestion (Begley et al., 2006). Consequently, BSH activity has significant impact on host physiology by disturbing CBA-mediated fat metabolism and endocrine functions (Begley et al., 2006; Jones et al., 2008).

Feighner and Dashkevicz (1987) provided early evidence that antibiotic feed additives affect the transformation potential and hydrolysis activity of BSHs from intestinal contents of poultry. By keying in on a more specific aspect of lipid metabolism, Knarreborg etal. (2004) demonstrated an enhanced bioavailability of $\alpha$-tocopheryl (alpha-tocopherol) acetate in broilers given AGPs, and this was attributed to a reduced concentration of unconjugated bile salts. Furthermore, Guban etal. (2006) correlated dietary supplementation of AGPs to improved weight gain and fat digestibility in broilers, decreased population levels of Lactobacillus salivarius, and a reduced pool of deconjugated bile salts. Based on these discoveries, the growthpromoting effect of AGPs likely is partly attributed to the reduced BSH activity, and thus the improvement of host lipid metabolism.

\section{RESPONSE OF INTESTINAL MICROBIOTA TO AGP}

With the aid of culture-independent molecular approaches, the investigations of the effect of AGPs on intestinal microbiota have been initiated in different food animals, including poultry and swine, which greatly improves our understanding of intestinal 
microbiota changes in response to AGPs (Engberg et al., 2000; Knarreborg et al., 2002; Collier et al., 2003; Dumonceaux et al., 2006; Guban etal., 2006; Wise and Siragusa, 2007; Zhou et al., 2007; Danzeisen et al., 2011; Kim et al., 2012; Lin et al., 2013). As expected, oral administration of low-dose antibiotics affected diversity and relative abundance of gut microbiota in all these studies. However, based on the findings from these molecular ecology studies, it is challenging to definitively link specific bacterial populations to the enhanced growth performance due to AGP usage. Interestingly, independent studies (Engberg etal., 2000; Knarreborg etal., 2002; Dumonceaux etal., 2006; Guban etal., 2006; Zhou etal., 2007; Danzeisen etal., 2011; Lin etal., 2013) showed that AGP usage significantly reduced the population of Lactobacillus species, a major bacterial choice for probiotics development; in particular, population of $L$. salivarius, the dominant lactic acid bacterium present in the chicken intestine, was reduced in response to AGP treatment. Since Lactobacillus populations are major BSHproducers in small intestine (Begley etal., 2006), this interestingly bacterial shift due to AGP usage is consistent with the reduced BSH activity as described above. Taken together, these studies indicate that the AGP-mediated body weight gain in food animal is inversely related to the activity of $\mathrm{BSH}$ enzymes and the abundance of corresponding Lactobacilli producers.

\section{CHARACTERISTICS OF BSH}

Bacterial BSH is a member of the choloylglycine hydrolase family of enzymes and is predominantly associated with gastrointestinal bacteria of both humans and animals. Additionally, it is classified as an N-terminal nucleophilic hydrolase and can recognize substrate at both the amino acid conjugate or steroid nucleus (Patel et al., 2010). BSH is particularly abundant in lactic acid fermenting probiotic strains like Lactobacilli and Bifidobacteria (Begley et al., 2006). BSH enzymes display either narrow or broad substrate specificity; most BSH enzymes from lactic acid bacteria showed higher catalytic abilities to hydrolyze glyco-CBAs than tauro-CBAs (Begley et al., 2006). Despite recent significant progress in the characterization of diverse BSH enzymes, research on BSH is still in its infancy (Patel et al., 2010). The natural functions of BSH enzymes for bacteria themselves are still not clear (Begley et al., 2006). One hypothesis is that BSH activity confers Lactobacilli tolerance to bile in the intestine (Begley et al., 2006). However, there are contradictory reports about the correlation between bile tolerance and BSH activity in intestinal bacteria (Begley et al., 2006). For example, production of BSH does not determine bile resistance level in L. salivarius, the dominant Lactobacillus species present in the chicken intestine (Fang et al., 2009). Regardless the natural function of BSH for its bacterial producer, it has been increasingly recognized that intestinal $\mathrm{BSH}$ plays an important role in host lipid metabolism and energy harvest (Begley et al., 2006; Jones et al., 2008). Recent probiotics studies have already shown that oral administration of BSH-producing Lactobacilli could affect lipid metabolism, consequently lowering cholesterol level in humans (Jones et al., 2012), rats (Kumar et al., 2011), and pigs (De et al., 1998), which is likely mediated through BSH activity. In the future, more functional, genomic, and microbiological studies are needed to better understand the role of BSH in the symbiosis relationship between gut microbiota and host.

\section{PRODUCTION OF BSH BY PROBIOTICS: A NEGATIVE TRAIT FROM ANIMAL PRODUCTION PERSPECTIVES?}

It is no doubt that dietary probiotics, the normal commensal microorganisms, could exert various beneficial effects on food animals. However, probiotics do impose a variety of potential costs (or detrimental effects) to the animal host as well, which include the production of toxic metabolites, decreased fat digestibility due to production of $\mathrm{BSH}$, and the increase of mucus secretion and gut epithelial cell turn-over (Gaskins et al., 2002; Dibner and Richards, 2004, 2005). Due to such opposite impact of probiotics on the host, it is not surprising that inconsistent results on growth performance of poultry have been observed following probiotic administration (Perumalla etal., 2011). In particular, results available from the literature on probiotic treatments often appear to be contradictory. Lactobacilli have been a major bacterial choice for probiotic development in poultry. In general, dietary probiotic supplementation in chicken increases intestinal populations of Lactobacilli (Smirnov et al., 2005). Since Lactobacilli are dominant BSH-producers in the intestine, dietary Lactobacilli treatment may negatively affect lipid metabolism and energy harvest, consequently imposing negative impact on body weight gain. Recently, two research groups (Mountzouris et al., 2010; Sharifi et al., 2012) have reported that probiotic supplementation to diets significantly reduced body weight gain, fat digestibility, and feed conversion in broilers; in these studies, two different commercially available probiotics with a mixture of various organisms (Protexin and PoultryStar) were used. Based on these findings, the authors have proposed that the detrimental effects of the probiotics on chicken growth are likely attributed to the production of intestinal BSH by Lactobacilli (Mountzouris et al., 2010; Sharifi et al., 2012). Therefore, overall beneficial effects associated with specific probiotics should be carefully evaluated. Understanding the science of potential negative traits of probiotics can help us develop "negative-traits-mitigation" strategy (e.g., BSH inhibitors) to optimize probiotic products for enhanced growth performance of food animals and profitability of feed additive industry.

\section{DISCOVERY OF BSH INHIBITORS}

Based on the information reviewed above, BSH inhibitors are promising alternatives to AGP for enhanced production of food animals. This hypothesis has been partly supported by our recent study (Wang et al., 2012) in which a BSH enzyme with broad substrate specificity from a chicken $L$. salivarius strain was characterized. Examination of a panel of dietary compounds identified copper and zinc compounds as potent BSH inhibitor (Wang et al., 2012); notably, copper and/or zinc have been used at high concentrations to aid in feed efficiency and growth promotion in poultry (Ewing et al., 1998; Miles et al., 1998; Arias and Koutsos, 2006) and swine (Smith et al., 1997; Hill et al., 2000; Armstrong et al., 2004). However, long-term use of high doses of copper or zinc in animal feed has raised some serious concerns, such as copper/zinc toxicosis and environmental contamination. Therefore, discovery of potent, safe, and cost-effective BSH inhibitors is highly warranted. 
In our recent study (Smith et al., 2014), a rapid and convenient high-throughput screening (HTS) system was developed and has been successfully used for identification of BSH inhibitors. This HTS strategy is based on the unique feature of BSH enzyme: hydrolysis of soluble unconjugated bile salts by BSH generates insoluble unconjugated bile salts that could form significant precipitations (Smith et al., 2014). After optimizing various screening conditions, a pilot HTS was performed using a small compound library comprised of 2,240 diverse compounds, leading to the identification of several promising BSH inhibitors with potential as alternatives to AGPs, such as riboflavin and phenethyl caffeate (Smith etal., 2014). In the future, larger scale HTS may reveal more novel BSH inhibitors. In addition, comprehensive animal trials are needed to determine the effect of "champion" BSH inhibitors on growth performance of food animals.

Interestingly, this HTS study also identified a panel of antibiotics as BSH inhibitor, such as various tetracycline antibiotics and roxarsone that have been widely used as AGPs in food animals (Smith et al., 2014). This unexpected finding suggests a new mode of action of low-dose antibiotics for promoting animal growth: some AGPs may exert their growth-promoting effect on food animals by direct inhibition of intestinal BSH enzymes for enhanced lipid metabolism and energy harvest. However, some AGPs, such as bacitracin that has been widely used in poultry industry (Chapman and Johnson, 2002), only displayed low or little inhibitory effect on BSH activity (Smith et al., 2014). The study by Smith etal. (2014) further showed the complexity of the modes of action of AGPs and provided new insights into the interactions between low-dose antibiotics and gut microbiome.

\section{CONCLUSIONS AND FUTURE DIRECTIONS}

Independent studies have shown the link between usage of AGPs and reduced BSH activity as well as reduced population of some Lactobacillus species, the major BSH-producers, in the intestine of food animals. In light of these findings, BSH enzyme is a promising microbiome target for developing novel alternatives to AGPs for enhancing the productivity and sustainability of food animals. Recent characterization of a broad-spectrum BSH from L. salivarius (Wang et al., 2012) and development of an efficient HTS system for discovery of BSH inhibitors (Smith et al., 2014) have laid a solid foundation for us to develop BSH inhibitors-based feed additives to replace AGPs. In addition, the findings from screening of BSH inhibitors (Smith et al., 2014) suggest a new mode of action of low-dose antibiotics (direct inhibition of intestinal BSH) for promoting animal growth. In the future, large animal trials are needed to determine the effects of BSH inhibitors on growth performance of food animals. Given that research on BSH is still in its infancy, research on BSH ecology of BSH enzymes in the intestine and the role of BSH in host physiology is also highly warranted in the future.

\section{ACKNOWLEDGMENTS}

Work in our laboratory was supported by University of Tennessee AgResearch Innovation Grant and University of Tennessee Research Foundation Technology Maturation Fund.

\section{REFERENCES}

Arias, V. J., and Koutsos, E. A. (2006). Effects of copper source and level on intestinal physiology and growth of broiler chickens. Poult. Sci. 85, 999-1007.

Armstrong, T. A., Cook, D. R., Ward, M. M., Williams, C. M., and Spears, J. W. (2004). Effect of dietary copper source (cupric citrate and cupric sulfate) and concentration on growth performance and fecal copper excretion in weanling pigs. J. Anim. Sci. 82, 1234-1240.

Begley, M., Hill, C., and Gahan, C. G. (2006). Bile salt hydrolase activity in probiotics. Appl. Environ. Microbiol. 72, 1729-1738. doi: 10.1128/AEM.72.3.1729-17 38.2006

Chapman, H. D., and Johnson, Z. B. (2002). Use of antibiotics and roxarsone in broiler chickens in the USA: analysis for the years 1995 to 2000. Poult. Sci. 81, 356-364.

Collier, C. T., Smiricky-Tjardes, M. R., Albin, D. M., Wubben, J. E., Gabert, V. M., Deplancke, B. etal. (2003). Molecular ecological analysis of porcine ileal microbiota responses to antimicrobial growth promoters. J. Anim. Sci. 81, 30353045.

Danzeisen, J. L., Kim, H. B., Isaacson, R. E., Tu, Z. J., and Johnson, T. J. (2011). Modulations of the chicken cecal microbiome and metagenome in response to anticoccidial and growth promoter treatment. PLoS ONE 6:e27949. doi: 10.1371/journal.pone.0027949

De, S., I., De Boever, P., and Verstraete, W. (1998). Cholesterol lowering in pigs through enhanced bacterial bile salt hydrolase activity. Br. J. Nutr. 79, 185-194. doi: 10.1079/BJN19980030

Dibner, J. J., and Richards, J. D. (2004). The digestive system: challenges and opportunities. J. Appl. Poult. Res. 13, 86-93.

Dibner, J. J., and Richards, J. D. (2005). Antibiotic growth promoters in agriculture: history and mode of action. Poult. Sci. 84, 634-643.

Dumonceaux, T. J., Hill, J. E., Hemmingsen, S. M., and Van Kessel, A. G. (2006). Characterization of intestinal microbiota and response to dietary virginiamycin supplementation in the broiler chicken. Appl. Environ. Microbiol. 72, 2815-2823. doi: 10.1128/AEM.72.4.2815-2823.2006

Engberg, R. M., Hedemann, M. S., Leser, T. D., and Jensen, B. B. (2000). Effect of zinc bacitracin and salinomycin on intestinal microflora and performance of broilers. Poult. Sci. 79, 1311-1319.

Ewing, H. P., Pesti, G. M., Bakalli, R. I., and Menten, J. F. M. (1998). Studies on the feeding of cupric sulfate pentahydrate, cupric citrate, and copper oxychloride to broiler chickens. Poult. Sci. 77, 445-448.

Fang, F., Li, Y., Bumann, M., Raftis, E. J., Casey, P. G., Cooney, J. C. et al. (2009). Allelic variation of bile salt hydrolase genes in Lactobacillus salivarius does not determine bile resistance levels. J. Bacteriol. 191, 5743-5757. doi: 10.1128/JB. 00506-09

Feighner, S. D., and Dashkevicz, M. P. (1987). Subtherapeutic levels of antibiotics in poultry feeds and their effects on weight gain, feed efficiency, and bacterial cholyltaurine hydrolase activity. Appl. Environ. Microbiol. 53, 331-336.

Gaskins, H. R., Collier, C. T., and Anderson, D. B. (2002). Antibiotics as growth promotants: mode of action. Anim. Biotechnol. 13, 29-42. doi: 10.1081/ABIO120005768

Guban, J., Korver, D. R., Allison, G. E., and Tannock, G. W. (2006). Relationship of dietary antimicrobial drug administration with broiler performance, decreased population levels of Lactobacillus salivarius, and reduced bile salt deconjugation in the ileum of broiler chickens. Poult. Sci. 85, 2186-2194.

Hill, G. M., Cromwell, G. L., Crenshaw, T. D., Dove, C. R., Ewan, R. C., Knabe, D. A. et al. (2000). Growth promotion effects and plasma changes from feeding high dietary concentrations of zinc and copper to weanling pigs (regional study). J. Anim. Sci. 78, 1010-1016.

Jones, B. V., Begley, M., Hill, C., Gahan, C. G. M., and Marchesi, J. R. (2008). Functional and comparative metagenomic analysis of bile salt hydrolase activity in the human gut microbiome. Proc. Natl. Acad. Sci. U.S.A. 105, 13580-13585. doi: 10.1073/pnas.0804437105

Jones, M. L., Martoni, C. J., Parent, M., and Prakash, S. (2012). Cholesterol-lowering efficacy of a microencapsulated bile salt hydrolase-active Lactobacillus reuteri NCIMB 30242 yoghurt formulation in hypercholesterolaemic adults. Br. J. Nutr. 107, 1505-1513. doi: 10.1017/S0007114511004703

Kim, H. B., Borewicz, K., White, B. A., Singer, R. S., Sreevatsan, S., Tu, Z. J., et al. (2012). Microbial shifts in the swine distal gut in response to the treatment with antimicrobial growth promoter, tylosin. Proc. Natl. Acad. Sci. U.S.A. 109, 15485-15490. doi: 10.1073/pnas. 1205147109 
Knarreborg, A., Lauridsen, C., Engberg, R. M., and Jensen, S. K. (2004). Dietary antibiotic growth promoters enhance the bioavailability of alpha-tocopheryl acetate in broilers by altering lipid absorption. J. Nutr. 134, 1487-1492.

Knarreborg, A., Simon, M. A., Engberg, R. M., Jensen, B. B., and Tannock, G. W. (2002). Effects of dietary fat source and subtherapeutic levels of antibiotic on the bacterial community in the ileum of broiler chickens at various ages. Appl. Environ. Microbiol. 68, 5918-5924. doi: 10.1128/AEM.68.12.5918-5924.2002

Kumar, R., Grover, S., and Batish, V. K. (2011). Hypocholesterolaemic effect of dietary inclusion of two putative probiotic bile salt hydrolase-producing Lactobacillus plantarum strains in Sprague-Dawley rats. Br. J. Nutr. 105, 561-573. doi: 10.1017/S0007114510003740

Lin, J., Hunkapiller, A. A., Layton, A. C., Chang, Y. J., and Robbins, K. R. (2013). Response of intestinal microbiota to antibiotic growth promoters in chickens. Foodborne Pathog. Dis. 10, 331-337. doi: 10.1089/fpd.2012.1348

Miles, R. D., O’Keefe, S. F., Henry, P. R., Ammerman, C. B., and Luo, X. G. (1998). The effect of dietary supplementation with copper sulfate or tribasic copper chloride on broiler performance, relative copper bioavailability, and dietary prooxidant activity. Poult. Sci. 77, 416-425.

Mountzouris, K. C., Tsitrsikos, P., Palamidi, I., Arvaniti, A., Mohnl, M., Schatzmayr, G., et al. (2010). Effects of probiotic inclusion levels in broiler nutrition on growth performance, nutrient digestibility, plasma immunoglobulins, and cecal microflora composition. Poult. Sci. 89, 58-67. doi: 10.3382/ps.2009-00308

Patel, A. K., Singhania, R. R., Pandey, A., and Chincholkar, S. B. (2010). Probiotic bile salt hydrolase: current developments and perspectives. Appl. Biochem. Biotechnol. 162, 166-180. doi: 10.1007/s12010-009-8738-1

Perumalla, A. V. S., Hettiarachchy, N. N., and Ricke, S. C. (2011) "Current perspectives on probiotics in poultry preharvest food safety," in Direct-Fed Microbials and Probiotics for Animals: Science and Mechansims of Action, eds T. R. Callaway and S. C. Ricke (New York: Springer), 89-120.

Sharifi, S. D., Dibamehr, A., Lotfollahian, H., and Baurhoo, B. (2012). Effects of flavomycin and probiotic supplementation to diets containing different sources of fat on growth performance, intestinal morphology, apparent metabolizable energy, and fat digestibility in broiler chickens. Poult. Sci. 91, 918-927. doi: 10.3382/ps.2011-01844

Smirnov, A., Perez, R., Amit-Romach, E., Sklan, D., and Uni, Z. (2005). Mucin dynamics and microbial populations in chicken small intestine are changed by dietary probiotic and antibiotic growth promoter supplementation. J. Nutr. 135, 187-192.

Smith, J. W., Tokach, M. D., Goodband, R. D., Nelssen, J. L., and Richert, B. T. (1997). Effects of the interrelationship between zinc oxide and copper sulfate on growth performance of early-weaned pigs. J. Anim. Sci. 75, 1861-1866.
Smith, K., Zeng, X., and Lin, J. (2014). Discovery of bile salt hydrolase inhibitors using an efficient high-throughput screening system. PLoS ONE 9:e85344. doi: 10.1371/jounal.pone.0085344

Turnidge, J. (2004). Antibiotic use in animals-prejudices, perceptions and realities. J. Antimicrob. Chemother. 53, 26-27. doi: 10.1093/jac/dkg493

Wang, Z., Zeng, X., Mo, Y., Smith, K., Guo, Y., and Lin, J. (2012). Identification and characterization of a bile salt hydrolase from Lactobacillus salivarius for development of novel alternatives to antibiotic growth promoters. Appl. Environ. Microbiol. 78, 8795-8802. doi: 10.1128/AEM. 02519-12

Wegener, H. C. (2003). Antibiotics in animal feed and their role in resistance development. Curr. Opin. Microbiol. 6, 439-445. doi: 10.1016/j.mib.2003. 09.009

Wise, M. G., and Siragusa, G. R. (2007). Quantitative analysis of the intestinal bacterial community in one- to three-week-old commercially reared broiler chickens fed conventional or antibiotic-free vegetable-based diets. J. Appl. Microbiol. 102, 1138-1149. doi: 10.1111/j.1365-2672.2006. 03153.x

Zhou, H., Gong, J., Brisbin, J. T., Yu, H., Sanei, B., Sabour, P., et al. (2007). Appropriate chicken sample size for identifying the composition of broiler intestinal microbiota affected by dietary antibiotics, using the polymerase chain reactiondenaturing gradient gel electrophoresis technique. Poult. Sci. 86, 2541-2549.doi: $10.3382 /$ ps.2007-00267

Conflict of Interest Statement: The author declares that the research was conducted in the absence of any commercial or financial relationships that could be construed as a potential conflict of interest.

Received: 06 December 2013; accepted: 17 January 2014; published online: 11 February 2014.

Citation: Lin J (2014) Antibiotic growth promoters enhance animal production by targeting intestinal bile salt hydrolase and its producers. Front. Microbiol. 5:33. doi: 10.3389/fmicb.2014.00033

This article was submitted to Antimicrobials, Resistance and Chemotherapy, a section of the journal Frontiers in Microbiology.

Copyright (c) 2014 Lin. This is an open-access article distributed under the terms of the Creative Commons Attribution License (CC BY). The use, distribution or reproduction in other forums is permitted, provided the original author(s) or licensor are credited and that the original publication in this journal is cited, in accordance with accepted academic practice. No use, distribution or reproduction is permitted which does not comply with these terms. 